Article

\title{
Rhodium-Catalyzed Linear Codimerization and Cycloaddition of Ketenes with Alkynes
}

\section{Teruyuki Kondo ${ }^{1,2, *}$, Masatsugu Niimi ${ }^{2}$, Yuki Yoshida ${ }^{2}$, Kenji Wada ${ }^{2}$, Take-aki Mitsudo ${ }^{2}$, Yu Kimura ${ }^{1}$ and Akio Toshimitsu ${ }^{3}$}

1 Advanced Biomedical Engineering Research Unit, Kyoto University, Katsura, Nishikyo-ku, Kyoto 615-8510, Japan; E-Mail: ykimura@scl.kyoto-u.ac.jp (Y.K.)

2 Department of Energy and Hydrocarbon Chemistry, Graduate School of Engineering, Kyoto University, Katsura, Nishikyo-ku, Kyoto 615-8510, Japan;

E-Mails: Masatsugu_Niimi@jsr.co.jp (M.N.); yoshida.y@ky3.ecs.kyoto-u.ac.jp (Y.Y.); wadaken@scl.kyoto-u.ac.jp (K.W.); mitsudo@tech.email.ne.jp (T.M.)

3 Office of Society-Academia Collaboration for Innovation, Kyoto University, Yoshida-Honmachi, Sakyo-ku, Kyoto 606-8501, Japan; E-Mail: akiot@saci.kyoto-u.ac.jp (A.T.)

* Author to whom correspondence should be addressed; E-Mail: teruyuki@scl.kyoto-u.ac.jp; Tel.: +81-75-383-7055; Fax: +81-75-383-2805.

Received: 11 May 2010; in revised form: 25 May 2010 / Accepted: 7 June 2010 /

Published: 9 June 2010

\begin{abstract}
A novel rhodium-catalyzed linear codimerization of alkyl phenyl ketenes with internal alkynes to dienones and a novel synthesis of furans by an unusual cycloaddition of diaryl ketenes with internal alkynes have been developed. These reactions proceed smoothly with the same rhodium catalyst, $\mathrm{RhCl}\left(\mathrm{PPh}_{3}\right)_{3}$, and are highly dependent on the structure and reactivity of the starting ketenes.
\end{abstract}

Keywords: rhodium; catalyst; ketene; alkyne; codimerization; cycloaddition

\section{Introduction}

Ketenes are very important intermediates in the field of organic synthesis [1-3], and much attention has been focused on the ketene-metal complexes [4]. In general, ketenes coordinate to transition-metal complexes in two ways: 1) coordination through a $\mathrm{C}=\mathrm{C}$ bond in ketenes [5], and 2) coordination 
through a $\mathrm{C}=\mathrm{O}$ bond in ketenes [6-10]. If these coordination modes can be controlled through the selection of ketenes in transition-metal catalysis, completely different methods for the construction of novel organic molecules could be developed according to the structure and reactivity of ketenes using the same transition-metal catalyst.

We have previously developed a ruthenium-catalyzed synthesis of pyranopyrandiones by the ringopening carbonylation of cyclopropenones [11] and a novel synthesis of 2-pyranones by the ruthenium- or rhodium catalyzed ring-opening dimerization of cyclobutenones [12], as well as a rhodium-catalyzed synthesis of 2-substituted phenols from cyclobutenones and alkenes via cleavage of a carbon-carbon bond [13]. We propose that ( $\eta^{4}$-bisketene)- and ( $\eta^{4}$-vinylketene)metal complexes are important key intermediates in these reactions; however, there are still few examples of transitionmetal complex-catalyzed transformations of ketenes themselves [14-20]. Thus, we focused our attention on the development of novel reactions of ketenes with unsaturated compounds in the presence of ruthenium or rhodium catalysts, and recently developed rhodium-catalyzed decarbonylative coupling reactions of diphenyl ketene with 2-norbornenes and electron-deficient alkenes [21]. Then, the reactions of ketenes with alkynes were investigated in the presence of several transition-metal catalysts. After many trials, we developed the novel $\mathrm{RhCl}\left(\mathrm{PPh}_{3}\right)_{3}$-catalyzed linear codimerization of alkyl phenyl ketenes with internal alkynes and a novel synthesis of furans by the unusual $\mathrm{RhCl}\left(\mathrm{PPh}_{3}\right)_{3}$-catalyzed cycloaddition of diaryl ketenes with internal alkynes. In these reactions, the catalyst is the same but the products are completely different, depending on the structure and reactivity of the starting ketenes.

\section{Results and Discussion}

Treatment of alkyl phenyl ketenes 1a-c with internal alkynes 2 in the presence of 5 mol \% $\mathrm{RhCl}\left(\mathrm{PPh}_{3}\right)_{3}$ in mesitylene at $120{ }^{\circ} \mathrm{C}$ for $12 \mathrm{~h}$ under an argon atmosphere gave the corresponding dienones 3 in high yield with high stereoselectivity (Scheme 1). For example, $\mathrm{RhCl}\left(\mathrm{PPh}_{3}\right)_{3}$-catalyzed reaction of ethyl phenyl ketene (1a) with 3-hexyne (2a) gave only (2Z, 5E)-5-ethyl-3-phenylocta-2,5dien-4-one (3a) in 92\% yield, and no stereoisomers were obtained at all.

Scheme 1. Rhodium-catalyzed linear codimerization of ketenes with alkynes to dienones.

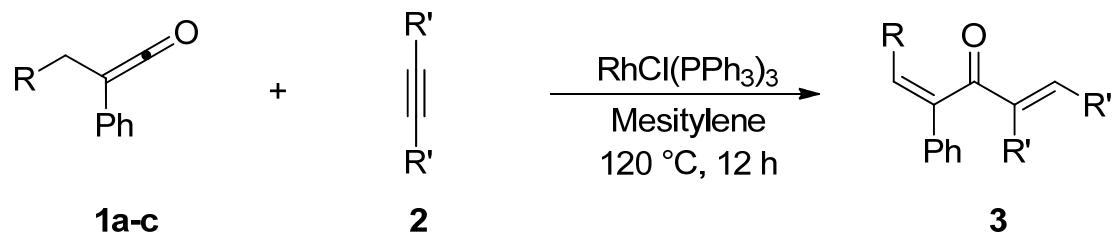

First, the catalytic activities of several transition-metal complexes were examined in the reaction of 1a with 2a, and the results are summarized in Table 1 . Among the catalysts examined, $\mathrm{RhCl}\left(\mathrm{PPh}_{3}\right)_{3}$ (3a, 92\%) showed the highest catalytic activity. $\mathrm{RhCl}(\mathrm{CO})\left(\mathrm{PPh}_{3}\right)_{2}(3 \mathbf{a}, 46 \%)$ and $\mathrm{RhCl}_{3} \cdot 3 \mathrm{H}_{2} \mathrm{O}(3 \mathbf{a}$, 26\%) also showed moderate catalytic activity; however, other rhodium complexes, such as $\mathrm{RhH}\left(\mathrm{PPh}_{3}\right)_{4}$ and $\mathrm{RhH}(\mathrm{CO})\left(\mathrm{PPh}_{3}\right)_{3}$, as well as ruthenium complexes, such as $\mathrm{RuCl}_{2}\left(\mathrm{PPh}_{3}\right)_{3}$, $\left[\mathrm{RuCl}_{2}(\mathrm{CO})_{3}\right]_{2}$, and $\mathrm{RuH}_{2}\left(\mathrm{PPh}_{3}\right)_{4}$, and an iridium complex, $\operatorname{IrCl}(\mathrm{CO})\left(\mathrm{PPh}_{3}\right)_{2}$, were totally ineffective, 
whereas $\mathrm{Pd}\left(\mathrm{PPh}_{3}\right)_{4}$ showed slight catalytic activity (3a, 29\%). An attempt to reduce the amount of $\mathrm{RhCl}\left(\mathrm{PPh}_{3}\right)_{3}$ catalyst from $5.0 \mathrm{~mol} \%$ to $2.0 \mathrm{~mol} \%$ resulted in vain (Entry 2 ).

Table 1. Catalytic activity of several transition-metal complexes in the reaction of 1a with 2a to 3a.

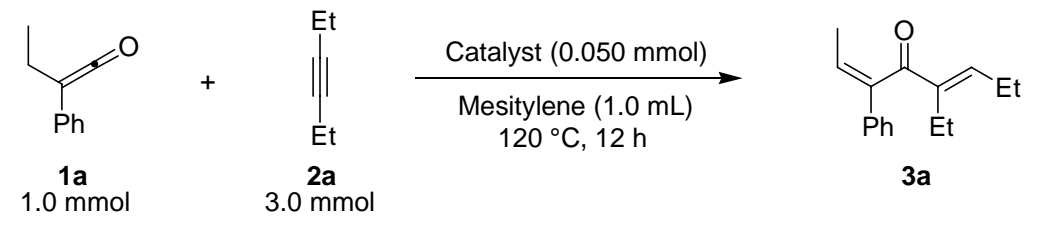

\begin{tabular}{ccc|ccc}
\hline Entry & Catalyst & Yield of 3a (\%) & Entry & Catalyst & Yield of 3a (\%) ${ }^{\text {a }}$ \\
\hline 1 & $\mathrm{RhCl}\left(\mathrm{PPh}_{3}\right)_{3}$ & 92 & 7 & $\mathrm{RuCl}_{2}\left(\mathrm{PPh}_{3}\right)_{3}$ & 1 \\
$2^{\mathrm{b}}$ & $\mathrm{RhCl}\left(\mathrm{PPh}_{3}\right)_{3}$ & 5 & $8^{\mathrm{c}}$ & {$\left[\mathrm{RuCl}_{2}(\mathrm{CO})_{3}\right]_{2}$} & 0 \\
3 & $\mathrm{RhCl}(\mathrm{CO})\left(\mathrm{PPh}_{3}\right)_{2}$ & 46 & 9 & $\mathrm{RuH}_{2}\left(\mathrm{PPh}_{3}\right)_{4}$ & 0 \\
4 & $\mathrm{RhCl}_{3} \cdot 3 \mathrm{H}_{2} \mathrm{O}$ & 26 & 10 & $\mathrm{Pd}_{\left(\mathrm{PPh}_{3}\right)_{4}}$ & 29 \\
5 & $\mathrm{RhH}\left(\mathrm{PPh}_{3}\right)_{4}$ & 2 & 11 & $\operatorname{IrCl}(\mathrm{CO})\left(\mathrm{PPh}_{3}\right)_{2}$ & 0 \\
6 & $\mathrm{RhH}(\mathrm{CO})\left(\mathrm{PPh}_{3}\right)_{3}$ & 0 & & & \\
\hline
\end{tabular}

${ }^{\mathrm{a}} \mathrm{GLC}$ yield; ${ }^{\mathrm{b}} \mathrm{RhCl}\left(\mathrm{PPh}_{3}\right)_{3}(2.0 \mathrm{~mol} \%, 0.020 \mathrm{mmol})$ for $40 \mathrm{~h} ;{ }^{\mathrm{c}}\left[\mathrm{RuCl}_{2}(\mathrm{CO})_{3}\right]_{2}(0.025 \mathrm{mmol})$.

Table 2. $\mathrm{RhCl}\left(\mathrm{PPh}_{3}\right)_{3}$-catalyzed linear codimerization of alkyl phenyl ketenes 1a-c with internal alkynes 2a,b to dienones 3a-d. ${ }^{\text {a }}$

Entry Ketene

${ }^{a}$ Ketene $(1.0 \mathrm{mmol})$, alkyne $(3.0 \mathrm{mmol}), \mathrm{RhCl}\left(\mathrm{PPh}_{3}\right)_{3}(0.050 \mathrm{mmol})$, and mesitylene $(1.0 \mathrm{~mL})$ at $120^{\circ} \mathrm{C}$ for $12 \mathrm{~h}$ under an argon atmosphere; ${ }^{\mathrm{b}}$ GLC yield.

The results obtained in the reaction of several alkyl phenyl ketenes 1a-c with alkynes 2a and $\mathbf{b}$ under the optimized reaction conditions are summarized in Table 2. Ethyl phenyl ketene (1a) reacted with 
6-dodecyne (2b) to give the corresponding dienone $3 \mathbf{b}$ in an isolated yield of $68 \%$ (Entry 2). As for ketenes, cycloalkyl phenyl ketenes, such as $\mathbf{1 b}$ and $\mathbf{1 c}$, also reacted with $\mathbf{2 a}$ to give the corresponding dienones, 3c and 3d, in isolated yields of 52\% and 40\%, respectively (Entries 3 and 4). Unfortunately, when terminal alkynes, such as phenylacetylene and 1-hexyne, were used in $\mathrm{RhCl}\left(\mathrm{PPh}_{3}\right)_{3}$-catalyzed reaction with ethyl phenyl ketene (1a), the corresponding dienones were obtained in low yield (below $10 \%$ ) together with various byproducts, probably due to the formation of a (vinylidene)Rh species.

In sharp contrast, treatment of diaryl ketenes 1d and e instead of alkyl phenyl ketenes 1a-c with internal alkynes 2 in the presence of the same $\mathrm{RhCl}\left(\mathrm{PPh}_{3}\right)_{3}$ catalyst $(5 \mathrm{~mol} \%)$ in mesitylene at $120{ }^{\circ} \mathrm{C}$ for $12 \mathrm{~h}$ under an argon atmosphere gave unusual cycloadducts, the furans $\mathbf{4}$, instead of dienones $\mathbf{3}$, in good to high yields (Scheme 2). The structure of furan 4a was confirmed by ${ }^{13} \mathrm{C}$ Inadequate NMR measurement (see Experimental, Figure 2).

Scheme 2. Rhodium-catalyzed cycloaddition of ketenes with alkynes to furans.

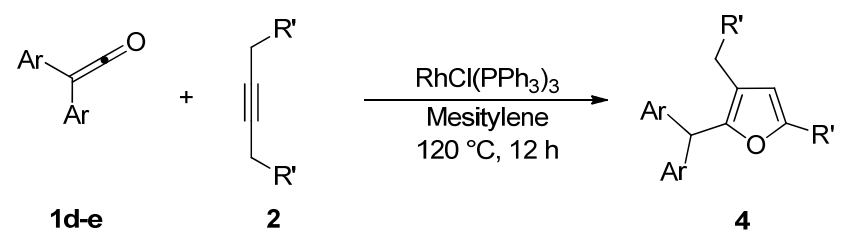

The catalytic activities of several transition-metal complexes were also examined in the reaction of diphenyl ketene (1d) with 3-hexyne (2a), and the results are summarized in Table 3. Among the catalysts examined, only $\mathrm{RhCl}\left(\mathrm{PPh}_{3}\right)_{3}$ showed catalytic activity (4a, 74\%). Other rhodium, ruthenium, iridium and palladium complexes were totally ineffective in the present reaction.

Table 3. Catalytic activity of several trasition-metal complexes in the reaction of $\mathbf{1 d}$ with $\mathbf{2 a}$ to $\mathbf{4 a}$.

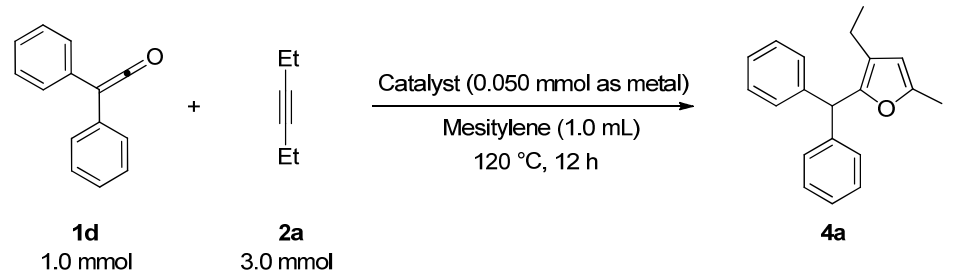

\begin{tabular}{ccc|ccc}
\hline Entry & Catalyst & Yield of 4a (\%) ${ }^{\text {a }}$ & Entry & Catalyst & Yield of 4a (\%) ${ }^{\mathbf{a}}$ \\
\hline 1 & $\mathrm{RhCl}\left(\mathrm{PPh}_{3}\right)_{3}$ & 74 & 7 & {$\left[\mathrm{Cp}^{*} \mathrm{RuCl}_{2}\right]_{2}$} & 0 \\
2 & $\mathrm{RhCl}(\mathrm{CO})\left(\mathrm{PPh}_{3}\right)_{2}$ & 2 & 8 & $\mathrm{RuCl}_{2}\left(\mathrm{PPh}_{3}\right)_{3}$ & 0 \\
3 & $\mathrm{RhCl} \cdot 3 \mathrm{H}_{2} \mathrm{O}$ & 0 & 9 & {$\left[\mathrm{RuCl}_{2}(\mathrm{CO})_{3}\right]_{2}$} & 0 \\
4 & {$[\mathrm{RhCl}(\mathrm{cod})]_{2}$} & 0 & 10 & $\mathrm{IrCl}(\mathrm{CO})\left(\mathrm{PPh}_{3}\right)_{2}$ & 0 \\
5 & {$\left[\mathrm{RhCl}\left(\mathrm{C}_{2} \mathrm{H}_{4}\right)_{2}\right]_{2}$} & 0 & 11 & $\mathrm{Pd}\left(\mathrm{PPh}_{3}\right)_{4}$ & 0 \\
6 & $\mathrm{RhH}\left(\mathrm{PPh}_{3}\right)_{4}$ & 0 & & & \\
\hline
\end{tabular}

${ }^{a}$ GLC yield. 
6-Dodecyne (2b), as well as 4-octyne (2c) and 5-decyne (2d), reacted with $\mathbf{1 d}$ to give the corresponding furans $\mathbf{4 b}$-d in moderate yields (Entries 2-4 in Table 4). As for ketenes, di(4-chlorophenyl) ketene (1e) also reacted with 2a to give the corresponding furan $4 \mathbf{e}$ in an isolated yield of $51 \%$ (Entry 5).

Table 4. $\mathrm{RhCl}\left(\mathrm{PPh}_{3}\right)_{3}$-catalyzed unusual cycloaddition of diaryl ketenes $\mathbf{1 d , e}$ with internal alkynes 2a-d to furans $\mathbf{4 a - e .}{ }^{\text {a }}$

Entry Ketene

${ }^{\mathrm{a}}$ Ketene $(1.0 \mathrm{mmol})$, alkyne $(3.0 \mathrm{mmol}), \mathrm{RhCl}\left(\mathrm{PPh}_{3}\right)_{3}(0.050 \mathrm{mmol})$, and mesitylene $(1.0 \mathrm{~mL})$ at $120{ }^{\circ} \mathrm{C}$ for $12 \mathrm{~h}$ under an argon atmosphere; ${ }^{\mathrm{b}} \mathrm{GLC}$ yield.

While the reaction mechanism is not yet clear, the possible mechanisms are illustrated in Schemes 3 and 4 . Scheme 3 shows a possible mechanism of the reaction of alkyl phenyl ketenes 1a-c with internal alkynes 2 to give dienones 3. We now believe that the initial step is the coordination of alkyl phenyl ketenes 1 to an active rhodium center through a $\mathrm{C}=\mathrm{C}$ bond in ketenes. Oxidative cyclization of alkyl phenyl ketenes 1a-c with alkynes 2 would give rhodacyclopentenone intermediates I [5]. Stereoselective $\beta$-hydrogen elimination, followed by reductive elimination, would give the corresponding dienones 3 stereoselectively. In addition, we now consider that a catalytically active species is a $\mathrm{Rh}(\mathrm{I})$ bearing a chloro ligand, and $\mathrm{RhCl}_{3} 3 \mathrm{H}_{2} \mathrm{O}$ would be reduced to a $\mathrm{Rh}(\mathrm{I})-\mathrm{Cl}$ species by crystal water to show some catalytic activity (Entry 4 in Table 1). 
Scheme 3. A possible mechanism of linear codimerization of alkyl phenyl ketenes 1a-c with internal alkynes 2 to give dienones 3 .

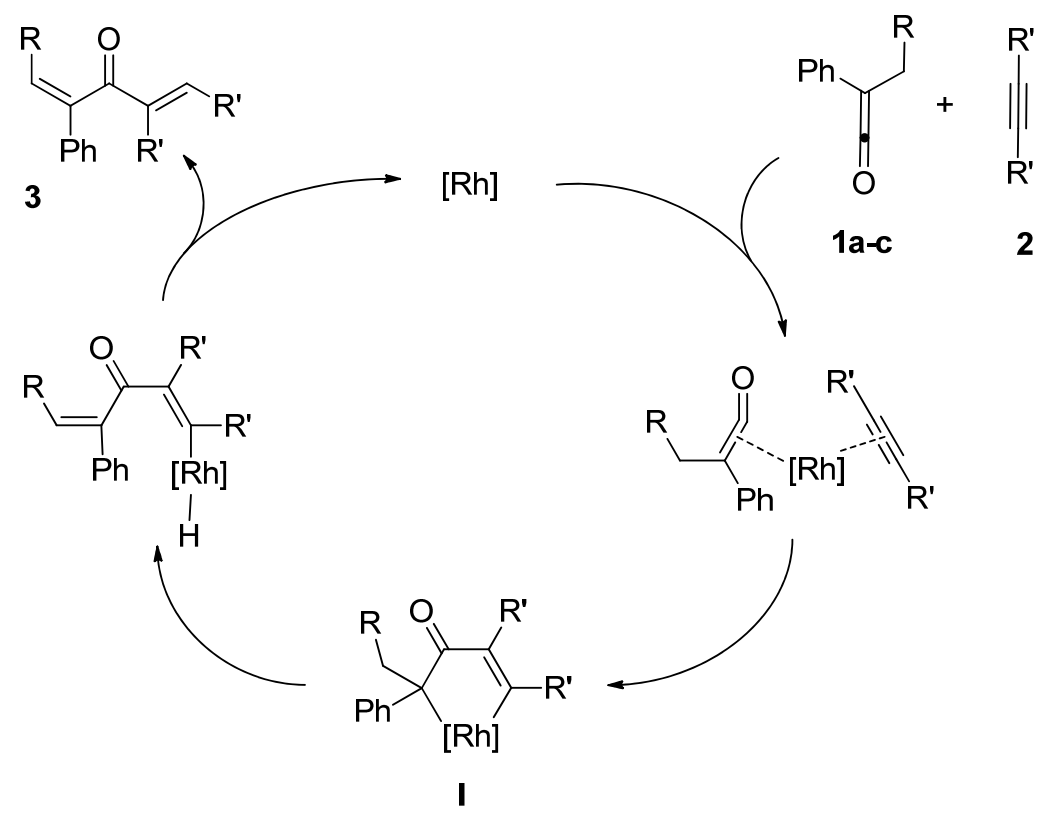

Scheme 4. A possible mechanism of the synthesis of furans $\mathbf{4}$ by unusual cycloaddition of diaryl ketenes 1d and $\mathbf{e}$ with internal alkynes $\mathbf{2}$.

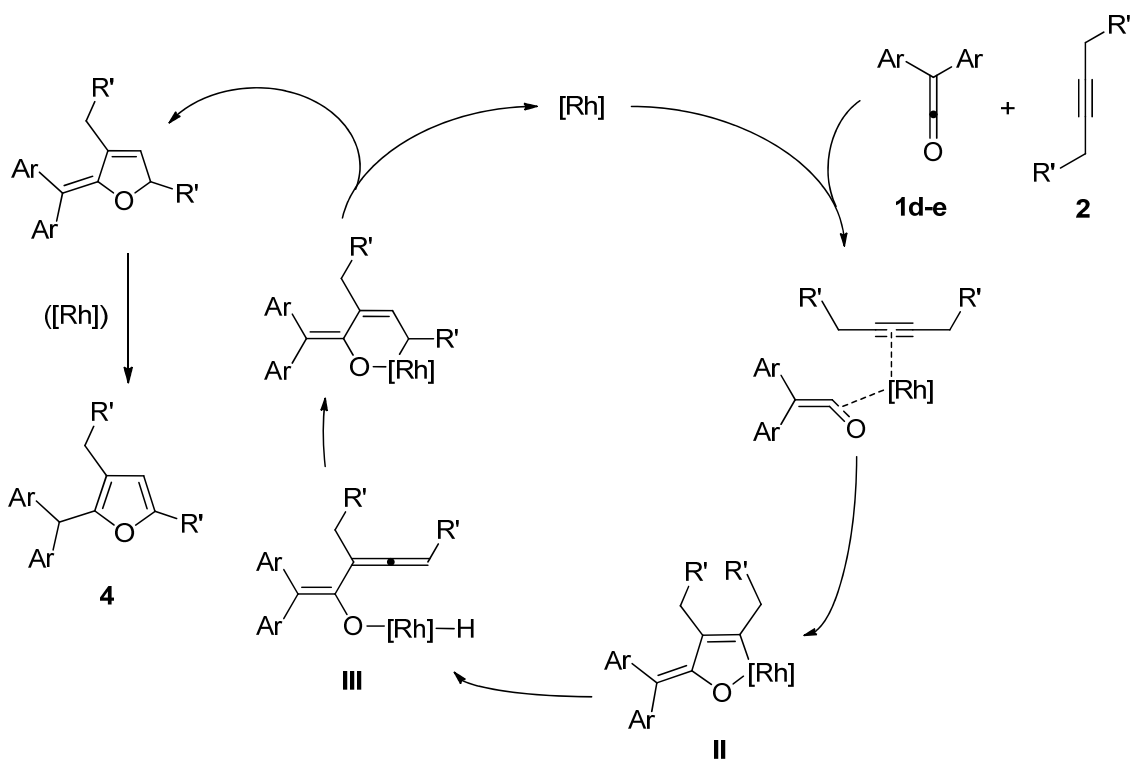

On the other hand, a possible mechanism for the reaction of diaryl ketenes $1 \mathbf{d}$ and $\mathbf{e}$ with internal alkynes 2 to furans 4 is shown in Scheme 4. In the synthesis of furans $\mathbf{4}$, the reaction starts from the coordination of diaryl ketenes to an active rhodium center through a $\mathrm{C}=\mathrm{O}$ bond in ketenes (not through a $\mathrm{C}=\mathrm{C}$ bond in ketenes). Oxidative cyclization of diaryl ketenes 1d, and $\mathbf{e}$ with alkynes 2 gives an oxametallacycle intermediate II [22-25]. $\beta$-Hydrogen elimination and insertion of an allenyl group in 
an intermediate III into a $\mathrm{Rh}-\mathrm{H}$ bond, followed by reductive elimination/isomerization, would give the desired furans 4.

\section{Experimental}

\subsection{General}

GLC analyses were carried out on a Shimadzu GC-18A gas chromatograph equipped with a glass column $(2.8 \mathrm{~mm}$ i.d. $\times 3 \mathrm{~m})$ packed with Silicone OV-17 (2\% on Chromosorb W(AW-DMCS), 60-80 mesh). Recycling preparative HPLC was performed with an LC-918 (Japan Analytical Industry Co. Ltd.) equipped with JAIGEL-1H and $2 \mathrm{H}$ columns (GPC) using $\mathrm{CHCl}_{3}$ as an eluent. ${ }^{1} \mathrm{H}-\mathrm{NMR}$ spectra were recorded at 300 or $400 \mathrm{MHz}$, and ${ }^{13} \mathrm{C}-\mathrm{NMR}$ spectra were recorded at 75 or $100 \mathrm{MHz}$. Samples were analyzed in $\mathrm{CDCl}_{3}$, and the chemical shift values are expressed relative to $\mathrm{Me}_{4} \mathrm{Si}$ as an internal standard. IR spectra were obtained on a Nicolet Impact 410 spectrometer. Elemental analyses were performed at the Microanalytical Center of Kyoto University.

\subsection{Materials}

Ketenes were synthesized as described in the literature [26,27]. Alkynes were obtained commercially and purified before use by standard procedures. $\mathrm{RhCl}_{3} \cdot 3 \mathrm{H}_{2} \mathrm{O},[\mathrm{RhCl}(\operatorname{cod})]_{2},\left[\mathrm{Cp}^{*} \mathrm{RhCl}_{2}\right]_{2}$, $\left[\mathrm{RuCl}_{2}(\mathrm{CO})_{3}\right]_{2}, \operatorname{IrCl}(\mathrm{CO})\left(\mathrm{PPh}_{3}\right)_{2}$, and $\mathrm{Pd}\left(\mathrm{PPh}_{3}\right)_{4}$ were obtained commercially and used without further purification. $\mathrm{RhCl}\left(\mathrm{PPh}_{3}\right)_{3}$ [28], $\mathrm{RhCl}(\mathrm{CO})\left(\mathrm{PPh}_{3}\right)_{2}$ [29], $\mathrm{RhH}\left(\mathrm{PPh}_{3}\right)_{4}$ [30], $\mathrm{RhH}(\mathrm{CO})\left(\mathrm{PPh}_{3}\right)_{3}$ [31], [ $\left.\mathrm{RhCl}\left(\mathrm{C}_{2} \mathrm{H}_{4}\right)_{2}\right]_{2}$ [32], $\mathrm{RuCl}_{2}\left(\mathrm{PPh}_{3}\right)_{3}$ [33], and $\mathrm{RuH}_{2}\left(\mathrm{PPh}_{3}\right)_{4}$ [34] were prepared as described in the literature.

\subsection{General procedure for the rhodium-catalyzed reaction of ketenes with alkynes to give dienones} and furans

A mixture of ketene 1 (1.0 mmol), alkyne $2(3.0 \mathrm{mmol}), \mathrm{RhCl}\left(\mathrm{PPh}_{3}\right)_{3}(0.050 \mathrm{mmol})$, and mesitylene $(1.0 \mathrm{~mL})$ was placed in a two-necked $20-\mathrm{mL}$ Pyrex flask equipped with a magnetic stirring bar and a reflux condenser under a flow of argon. The reaction was carried out at $120^{\circ} \mathrm{C}$ for $12 \mathrm{~h}$ with stirring. After the reaction mixture was cooled, the products were analyzed by GLC and isolated by Kugelrohr distillation followed by recycling preparative HPLC.

(2Z,5E)-5-Ethyl-3-phenylocta-2,5-dien-4-one (3a). Yellow liquid; b.p. $130^{\circ} \mathrm{C}$ (3.0 mmHg, Kugelrohr); IR $\left(\mathrm{cm}^{-1}\right) 1652(\mathrm{CO}) ;{ }^{1} \mathrm{H}-\mathrm{NMR}\left(\mathrm{CDCl}_{3}, 300 \mathrm{MHz}\right): \delta 0.98(\mathrm{t}, 3 \mathrm{H}, J=7.52 \mathrm{~Hz}, \mathrm{C} 8-\mathrm{H}), 1.03(\mathrm{t}, 3 \mathrm{H}$, $\left.J=7.52 \mathrm{~Hz}, 5-\mathrm{CH}_{2} \mathrm{CH}_{3}\right), 1.72(\mathrm{~d}, 3 \mathrm{H}, J=7.16 \mathrm{~Hz}, \mathrm{C} 1-\mathrm{H}), 2.24(\mathrm{dq}, 2 \mathrm{H}, J=7.52 \mathrm{~Hz}, \mathrm{C} 7-\mathrm{H}), 2.42$ (q, $\left.2 \mathrm{H}, J=7.52 \mathrm{~Hz}, 5-\mathrm{C}_{2} \mathrm{CH}_{3}\right), 6.15$ (q, $\left.1 \mathrm{H}, J=7.16 \mathrm{~Hz}, \mathrm{C} 2-\mathrm{H}\right), 6.64$ (t, $\left.1 \mathrm{H}, J=7.52 \mathrm{~Hz}, \mathrm{C} 6-\mathrm{H}\right), 7.19-$ 7.37 (m, 5H, 3-phenyl-H); ${ }^{13} \mathrm{C}-\mathrm{NMR}\left(\mathrm{CDCl}_{3}, 75 \mathrm{MHz}\right): \delta 13.09\left(5-\mathrm{CH}_{2} \mathrm{CH}_{3}\right), 13.79(\mathrm{C} 8), 15.33$ (C1), 18.12 (5- $\left.\mathrm{CH}_{2} \mathrm{CH}_{3}\right), 22.22$ (C7), 125.06 (C2), 125.55 (3-phenyl), 127.25 (3-phenyl), 128.48 (3-phenyl), 129.19 (C3 or C5), 142.85 (3-phenyl-C1), 145.41 (C3 or C5), 150.28 (C6), 200.61 (C4); MS (EI) m/z $228\left(\mathrm{M}^{+}\right)$. Anal. Calcd. for $\mathrm{C}_{16} \mathrm{H}_{20} \mathrm{O}$ : C 84.36, H 9.00. Found: C 84.14, H 8.83. A nuclear Overhauser enhancement (NOE) study was undertaken to determine the stereochemistry of dienone 3a. Irradiation 
of olefinic $\mathrm{CH}$ at $\delta 6.15 \mathrm{ppm}$ gave a $6.7 \% \mathrm{NOE}$ of the phenyl group at $\delta 7.26-7.28 \mathrm{ppm}$, while irradiation of $\mathrm{CH}_{2}$ in ethyl group at $\delta 2.24 \mathrm{ppm}$ showed 5.2\% $\mathrm{NOE}$ with $\mathrm{CH}_{3}$ in the other ethyl group at $\delta 1.03 \mathrm{ppm}$. The stereochemistry of 3a was therefore assigned to $2 Z$ and $5 E$ (Figure 1). The same method was used to determine the stereochemistry of $\mathbf{3 b}-\mathbf{d}$.

Figure 1. The NOE study of 3a.

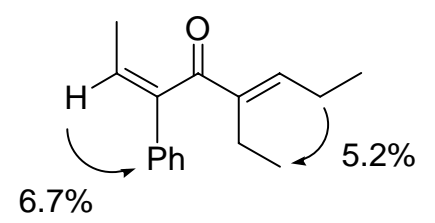

(2Z,5E)-5-Pentyl-3-phenylundeca-2,5-dien-4-one (3b). Colorless liquid; b.p. $140{ }^{\circ} \mathrm{C}(1.0 \mathrm{mmHg}$, Kugelrohr); IR ( $\left.\mathrm{cm}^{-1}\right)$ : 1652 (CO) ; ${ }^{1} \mathrm{H}-\mathrm{NMR}\left(\mathrm{CDCl}_{3}, 400 \mathrm{MHz}\right): \delta=0.84$ (t, 3H, C11-H), 0.89 (t, 3H, 5- $\left.\mathrm{CH}_{2}\left(\mathrm{CH}_{2}\right)_{3} \mathrm{C}_{3}\right), 1.17-1.35\left(\mathrm{~m}, 12 \mathrm{H}, 5-\mathrm{CH}_{2}\left(\mathrm{C}_{2}\right)_{3} \mathrm{CH}_{3} / \mathrm{C} 8-\mathrm{H} / \mathrm{C} 9-\mathrm{H} / \mathrm{C} 10-\mathrm{H}\right), 1.72$ (d, 3H, J = 7.16 Hz, C1-H), 2.21 (dt, 2H, C7-H), 2.37 (t, 2H, 5- $\left.\underline{\mathrm{H}}_{2}\left(\mathrm{CH}_{2}\right)_{3} \mathrm{CH}_{3}\right), 6.14$ (q, 1H, J = 7.16 Hz, C2-H), 6.66 (t, 1H, C6-H), 7.18-7.31 (m, 5H, 3-phenyl-H); ${ }^{13} \mathrm{C}-\mathrm{NMR}\left(\mathrm{CDCl}_{3}, 75 \mathrm{MHz}\right): \delta 13.93,14.04,15.38(\mathrm{C} 1)$, 22.40, 22.51, 24.97, 28.09, 28.61, 29.03, 31.89, 44.87, 125.09 (C2), 125.63 (3-phenyl), 127.14 (3phenyl), 128.61(3-phenyl), 134.58 (C3 or C5), 141.94 (3-phenyl-1), 144.80 (C3 or C5), 149.70 (C6), 200.62 (C4); MS (EI) m/z $312\left(\mathrm{M}^{+}\right)$.

(3E)-1-Cyclopentylidene-3-ethyl-1-phenylhex-3-en-2-one (3c). Yellow liquid; b.p. $140{ }^{\circ} \mathrm{C}(2.0 \mathrm{mmHg}$, Kugelrohr); IR ( $\left.\mathrm{cm}^{-1}\right) 1650$ (CO); ${ }^{1} \mathrm{H}-\mathrm{NMR}\left(\mathrm{CDCl}_{3}, 400 \mathrm{MHz}\right): \delta 0.96$ (t, 3H, J =7.52 Hz, C6-H), 0.98 (t, 3H, $J=7.52 \mathrm{~Hz}, 3-\mathrm{CH}_{2} \mathrm{CH}_{3}$ ), 1.61-1.71 (m, 4H, 1-cyclopentylidene-H), 2.21 (q, 2H, $J=7.52 \mathrm{~Hz}, 1$ cyclopentylidene-H), 2.30-2.46 (m, $6 \mathrm{H}, \quad 1$-cyclopentylidene-H/C5-H/3- $\left.\underline{\mathrm{H}}_{2} \mathrm{CH}_{3}\right), 6.63 \quad(\mathrm{t}, \quad 1 \mathrm{H}$, $J=7.52 \mathrm{~Hz}, \mathrm{C} 4-\mathrm{H}), 7.17-7.33\left(\mathrm{~m}, 5 \mathrm{H}, 1-\right.$ phenyl-H); ${ }^{13} \mathrm{C}-\mathrm{NMR}\left(\mathrm{CDCl}_{3}, 100 \mathrm{MHz}\right): \delta 13.31$ (3$\mathrm{CH}_{2} \mathrm{CH}_{3}$ ), 13.77 (C6), $18.51 \quad\left(3-\mathrm{CH}_{2} \mathrm{CH}_{3}\right), 22.19$ (C5), 26.34 (1-cyclopentylidene), 26.38 (1cyclopentylidene), 32.18 (1-cyclopentylidene), 32.39 (1-cyclopentylidene), 126.50 (1-phenyl), 127.82 (1-phenyl), 128.06 (1-phenyl), 132.91 (1-cyclopentylidene), 138.53 (C1 or C3), 142.08 (1-phenyl-1), 146.26 (C1 or C3), 148.48 (C4), 200.16(C2); MS (EI) m/z $268\left(\mathrm{M}^{+}\right)$.

(3E)-1-Cyclohexylidene-3-ethyl-1-phenylhex-3-en-2-one (3d). Yellow liquid; b.p. $160{ }^{\circ} \mathrm{C}(8.0 \mathrm{mmHg}$, Kugelrohr); IR ( $\left.\mathrm{cm}^{-1}\right) 1641$ (CO); ${ }^{1} \mathrm{H}-\mathrm{NMR}\left(\mathrm{CDCl}_{3}, 400 \mathrm{MHz}\right): \delta 0.91$ (t, 3H, C6-H), 1.04 (t, 3H, 3$\mathrm{CH}_{2} \underline{\mathrm{C}}_{3}$ ), 1.60 (br, 6H, 1-cyclohexylidene-H), 2.13 (br, 4H, 1-cyclohexylidene-H), 2.23 (br, 2H, C5$\mathrm{H}), 2.31\left(\mathrm{q}, 2 \mathrm{H}, J=7.32 \mathrm{~Hz}, 3-\mathrm{CH}_{2} \mathrm{CH}_{3}\right), 6.78$ (t, $\left.1 \mathrm{H}, J=7.32 \mathrm{~Hz}, \mathrm{C} 4-\mathrm{H}\right), 7.21-7.31$ (m, 5H, 1phenyl-H); ${ }^{13} \mathrm{C}-\mathrm{NMR}\left(\mathrm{CDCl}_{3}, 100 \mathrm{MHz}\right): \delta 13.62\left(3-\mathrm{CH}_{2} \mathrm{CH}_{3}\right), 13.79$ (C6), $18.46\left(3-\underline{\mathrm{CH}}_{2} \mathrm{CH}_{3}\right), 22.35$ (C5), 26.54 (1-cyclohexylidene), 27.96 (1-cyclohexylidene), 28.36 (1-cyclohexylidene), 30.90 (1cyclohexylidene), 32.88 (1-cyclohexylidene), 126.61 (1-phenyl), 128.77 (1-phenyl), 129.03 (1-phenyl), 133.32 (1-cyclohexylidene), 137.20 (C1 or C3), 140.50 (C1 or C3), 142.81 (1-phenyl-1), 148.96 (C4), 200.32(C2); MS (EI) m/z $282\left(\mathrm{M}^{+}\right)$. 
2-(Diphenylmethyl)-3-ethyl-5-methylfuran (4a). Yellow liquid; b.p. $135-145 \quad{ }^{\circ} \mathrm{C}(0.1 \mathrm{mmHg}$, Kugelrohr); ${ }^{1} \mathrm{H}-\mathrm{NMR}\left(\mathrm{CDCl}_{3}, 400 \mathrm{MHz}\right): \delta 1.05\left(\mathrm{t}, 3 \mathrm{H}, J=7.57 \mathrm{~Hz}, 3-\mathrm{CH}_{2} \mathrm{C}_{3}\right), 2.20\left(\mathrm{~s}, 3 \mathrm{H}, 5-\mathrm{CH}_{3}\right)$,

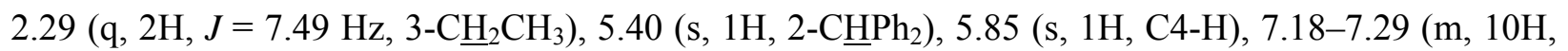
phenyl-H); ${ }^{13} \mathrm{C}-\mathrm{NMR}\left(\mathrm{CDCl}_{3}, 100 \mathrm{MHz}\right): \delta 13.85\left(5-\mathrm{CH}_{3}\right), 15.31\left(3-\mathrm{CH}_{2} \underline{\mathrm{CH}}_{3}\right), 18.24\left(3-\underline{C H}_{2} \mathrm{CH}_{3}\right)$, 48.43 (2- $\underline{\mathrm{CHPh}}$ ), 107.14 (C4), 123.09 (C3), 126.16 (phenyl), 128.24 (phenyl), 128.73 (phenyl), 142.30 (phenyl-1), 147.46 (C2), 150.22 (C5); MS (EI) m/z $276\left(\mathrm{M}^{+}\right)$. Anal. Calcd for $\mathrm{C}_{20} \mathrm{H}_{20} \mathrm{O}$ : C 86.92, $\mathrm{H}$ 7.29. Found: $\mathrm{C} 87.02, \mathrm{H} 7.29$. The relationship of the substituted group is confirmed by ${ }^{13} \mathrm{C}$ Inadequate NMR measurement (Figure 2).

2-(Diphenylmethyl)-5-n-butyl-3-n-pentylfuran (4b). Yellow liquid; b.p. $170{ }^{\circ} \mathrm{C}(0.1 \mathrm{mmHg}$, Kugelrohr); ${ }^{1} \mathrm{H}-\mathrm{NMR}\left(\mathrm{CDCl}_{3}, 400 \mathrm{MHz}\right): \delta 0.82(\mathrm{t}, 3 \mathrm{H}, J=6.84 \mathrm{~Hz}), 0.89(\mathrm{t}, 3 \mathrm{H}, J=7.32 \mathrm{~Hz}), 1.22(\mathrm{~m}, 6 \mathrm{H}, 3-$ $\left.\left(\mathrm{CH}_{2}\right)_{2}\left(\mathrm{CH}_{2}\right)_{2} \mathrm{CH}_{3} / 5-\left(\mathrm{CH}_{2}\right)_{2} \mathrm{C}_{2} \mathrm{CH}_{3}\right), 1.43$ (m, 2H, 3- $\left.\mathrm{CH}_{2} \underline{\mathrm{C}}_{2}\left(\mathrm{CH}_{2}\right)_{2} \mathrm{CH}_{3}\right), 1.56 \quad(\mathrm{~m}, \quad 2 \mathrm{H}, \quad 5-$ $\mathrm{CH}_{2} \mathrm{CH}_{2} \mathrm{CH}_{2} \mathrm{CH}_{3}$ ), 2.27 (dt, $\left.2 \mathrm{H}, J=2.12 \mathrm{~Hz}, 7.69 \mathrm{~Hz}, 3-\mathrm{CH}_{2}\left(\mathrm{CH}_{2}\right)_{3} \mathrm{CH}_{3}\right), 2.53$ (t, $2 \mathrm{H}, J=7.33 \mathrm{~Hz}, 5-$ $\left.\mathrm{C}_{2}\left(\mathrm{CH}_{2}\right)_{2} \mathrm{CH}_{3}\right), 5.37$ (s, 1H, 2-C $\left.\underline{H P h}_{2}\right), 5.82(\mathrm{~s}, 1 \mathrm{H}, \mathrm{C} 4-\mathrm{H}), 7.18-7.29\left(\mathrm{~m}, 10 \mathrm{H}\right.$, phenyl-H); ${ }^{13} \mathrm{C}-\mathrm{NMR}$ $\left(\mathrm{CDCl}_{3}, 100 \mathrm{MHz}\right): \delta 13.99,14.16,22.36,22.60,24.97\left(3-\mathrm{CH}_{2}\left(\mathrm{CH}_{2}\right)_{3} \mathrm{CH}_{3}\right), 27.86\left(5-\underline{\mathrm{CH}}_{2}\left(\mathrm{CH}_{2}\right)_{2} \mathrm{CH}_{3}\right)$, $30.20\left(5-\mathrm{CH}_{2} \mathrm{CH}_{2} \mathrm{CH}_{2} \mathrm{CH}_{3}\right), 30.34\left(3-\mathrm{CH}_{2} \mathrm{CH}_{2}\left(\mathrm{CH}_{2}\right)_{2} \mathrm{CH}_{3}\right), 31.04,48.38$ (2- $\left.\left.\underline{\mathrm{CHPh}}\right)_{2}\right), 106.54$ (C4), 121.41 (C3), 126.09 (phenyl), 128.01 (phenyl), 128.73 (phenyl), 142.48 (phenyl-1), 147.54 (C2), 154.63 (C5); MS (EI) m/z $360\left(\mathrm{M}^{+}\right)$.

Figure 2. ${ }^{13} \mathrm{C}$ Inadequate NMR spectrum of $\mathbf{4 a}$.

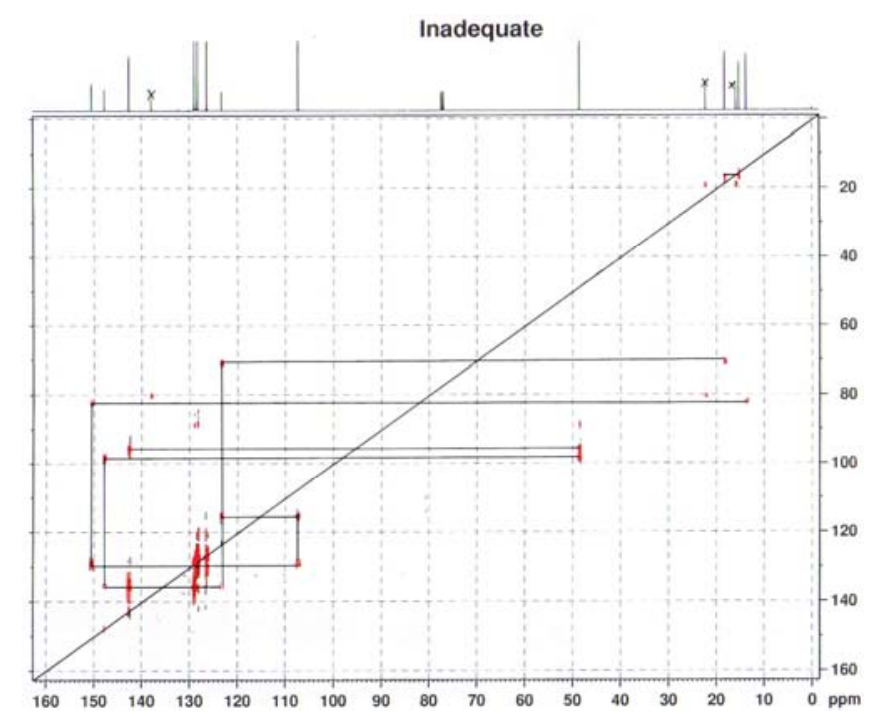

2-(Diphenylmethyl)-5-ethyl-3-n-propylfuran (4c). Yellow liquid; b.p. $140-150 \quad{ }^{\circ} \mathrm{C}(0.1 \mathrm{mmHg}$, Kugelrohr); ${ }^{1} \mathrm{H}-\mathrm{NMR}\left(\mathrm{CDCl}_{3}, 400 \mathrm{MHz}\right): \delta 0.85$ (t, $3 \mathrm{H}, J=7.33 \mathrm{~Hz}, 3-\mathrm{CH}_{2} \mathrm{CH}_{2} \mathrm{CH}_{3}$ ), 1.17 (t, $3 \mathrm{H}$, $J=7.33 \mathrm{~Hz}, 5-\mathrm{CH}_{2} \underline{\mathrm{C}}_{3}$ ), 1.47 (sixtet, $2 \mathrm{H}, J=7.42 \mathrm{~Hz}, 3-\mathrm{CH}_{2} \mathrm{C}_{2} \mathrm{CH}_{3}$ ), 2.26 (t, $2 \mathrm{H}, J=7.57 \mathrm{~Hz}, 3-$ $\mathrm{C}_{2} \mathrm{CH}_{2} \mathrm{CH}_{3}$ ), 2.56 (q, $\left.2 \mathrm{H}, J=7.53 \mathrm{~Hz}, 5-\underline{\mathrm{C}}_{2} \mathrm{CH}_{3}\right), 5.38$ (s, 1H, 2- $\left.\underline{\mathrm{HPh}}_{2}\right), 5.83(\mathrm{~s}, 1 \mathrm{H}, \mathrm{C} 4-\mathrm{H})$, 7.10-7.29 (m, 10H, phenyl-H); ${ }^{13} \mathrm{C}-\mathrm{NMR}\left(\mathrm{CDCl}_{3}, 100 \mathrm{MHz}\right): \delta 12.14\left(5-\mathrm{CH}_{2} \mathrm{CH}_{3}\right), 14.06$ (3$\left.\mathrm{CH}_{2} \mathrm{CH}_{2} \underline{\mathrm{CH}_{3}}\right), 21.46\left(5-\underline{\mathrm{CH}}_{2} \mathrm{CH}_{3}\right), 23.79\left(3-\mathrm{CH}_{2} \underline{\mathrm{CH}_{2}} \mathrm{CH}_{3}\right), 27.08\left(3-\underline{\mathrm{CH}_{2}} \mathrm{CH}_{2} \mathrm{CH}_{3}\right), 48.36\left(2-\underline{\mathrm{CHPh}_{2}}\right)$, 
105.86 (C4), 121.32 (C3), 126.18 (phenyl), 128.11 (phenyl), 128.82 (phenyl), 142.56 (phenyl-1), 147.81 (C2), 156.00 (C5); MS (EI) m/z $304\left(\mathrm{M}^{\dagger}\right)$.

2-(Diphenylmethyl)-3-n-butyl-5-n-propylfuran (4d). Yellow liquid; b.p. $150-160{ }^{\circ} \mathrm{C}(0.1 \mathrm{mmHg}$, Kugelrohr); ${ }^{1} \mathrm{H}-\mathrm{NMR}\left(\mathrm{CDCl}_{3}, 300 \mathrm{MHz}\right): \delta 0.84\left(\mathrm{t}, 3 \mathrm{H}, J=7.25 \mathrm{~Hz}, 3-\mathrm{CH}_{2}\left(\mathrm{CH}_{2}\right)_{2} \mathrm{C}_{3}\right), 0.91(\mathrm{t}, 3 \mathrm{H}$, $\left.J=7.52 \mathrm{~Hz}, 5-\left(\mathrm{CH}_{2}\right)_{2} \mathrm{CH}_{3}\right), 1.24\left(\mathrm{~m}, 4 \mathrm{H}, 3-\mathrm{CH}_{2}\left(\mathrm{C}_{2}\right)_{2} \mathrm{CH}_{3}\right), 1.59$ (sixtet, $2 \mathrm{H}, J=7.38 \mathrm{~Hz}, 5-$ $\left.\mathrm{CH}_{2} \underline{\mathrm{CH}}_{2} \mathrm{CH}_{3}\right), 2.27$ (t, $\left.2 \mathrm{H}, J=7.52 \mathrm{~Hz}, 3-\mathrm{CH}_{2}\left(\mathrm{CH}_{2}\right)_{2} \mathrm{CH}_{3}\right), 2.50\left(\mathrm{t}, 2 \mathrm{H}, J=7.43 \mathrm{~Hz}, 5-\mathrm{CH}_{2} \mathrm{CH}_{2} \mathrm{CH}_{3}\right)$, $5.37\left(\mathrm{~s}, 1 \mathrm{H}, 2-\mathrm{CHPh}_{2}\right), 5.83(\mathrm{~s}, 1 \mathrm{H}, \mathrm{C} 4-\mathrm{H}), 7.17-7.27\left(\mathrm{~m}, 10 \mathrm{H}\right.$, phenyl-H); ${ }^{13} \mathrm{C}-\mathrm{NMR}\left(\mathrm{CDCl}_{3}\right.$, $75 \mathrm{MHz}): \delta$ 13.71, 13.89, $21.39\left(5-\mathrm{CH}_{2} \mathrm{CH}_{2} \mathrm{CH}_{3}\right), 22.41,24.58 \quad\left(3-\mathrm{CH}_{2}\left(\mathrm{CH}_{2}\right)_{2} \mathrm{CH}_{3}\right), \quad 30.10$ $\underline{\mathrm{CH}}_{2} \mathrm{CH}_{2} \mathrm{CH}_{3}$ ), 32.72, 48.36 (2- $\underline{\mathrm{CHPh}}$ ), 106.79 (C4), 121.52 (C3), 126.23 (phenyl), 128.16 (phenyl), 128.88 (phenyl), 142.67 (phenyl-1), 147.77 (C2), 154.67 (C5); MS (EI) m/z $332\left(\mathrm{M}^{+}\right.$). Anal. Calcd for $\mathrm{C}_{24} \mathrm{H}_{26} \mathrm{O}: \mathrm{C}$ 86.70, H 8.43. Found: C 86.43, H 8.67.

2-[Bis(4-chloropheny)methyl]-3-ethyl-5-methylfuran (4e). Yellow liquid; b.p. $150{ }^{\circ} \mathrm{C}(3.0 \mathrm{mmHg}$, Kugelrohr); ${ }^{1} \mathrm{H}-\mathrm{NMR}\left(\mathrm{CDCl}_{3}, 300 \mathrm{MHz}\right): \delta 1.05\left(\mathrm{t}, 3 \mathrm{H}, J=7.57 \mathrm{~Hz}, 3-\mathrm{CH}_{2} \mathrm{C}_{3}\right), 2.20\left(\mathrm{~s}, 3 \mathrm{H}, 5-\underline{\mathrm{H}}_{3}\right)$, $2.28\left(\mathrm{q}, 2 \mathrm{H}, J=7.49 \mathrm{~Hz}, 3-\mathrm{C}_{2} \mathrm{CH}_{3}\right), 5.31\left(\mathrm{~s}, 1 \mathrm{H}, 2-\mathrm{C} \underline{\mathrm{H}}(p-\mathrm{ClPh})_{2}\right), 5.86(\mathrm{~s}, 1 \mathrm{H}, \mathrm{C} 4-\mathrm{H}), 7.06-7.31(\mathrm{~m}$, $8 \mathrm{H}$, phenyl-H); ${ }^{13} \mathrm{C}-\mathrm{NMR}\left(\mathrm{CDCl}_{3}, 75 \mathrm{MHz}\right): \delta 13.63\left(5-\mathrm{CH}_{3}\right), 15.10\left(3-\mathrm{CH}_{2} \underline{\mathrm{CH}}_{3}\right), 18.06\left(3-\underline{C H}_{2} \mathrm{CH}_{3}\right)$, $47.08\left(2-\underline{C H}(p-C l P h)_{2}\right), 107.35$ (C4), 128.47 (C3), 130.13 (phenyl), 132.03 (phenyl), 132.38 (phenyl), 140.56 (phenyl-1), 146.54 (C2), 150.87 (C5); MS (EI) m/z $344\left(\mathrm{M}^{+}\right)$.

\section{Conclusions}

In conclusion, we have developed a novel rhodium-catalyzed cross-coupling reaction of ketenes with alkynes. The different coordination modes of ketenes to rhodium, which highly depend on the structure and reactivity of the starting ketenes, realized the selective formation of totally different products, dienones and furans in the presence of the same rhodium catalyst, $\mathrm{RhCl}\left(\mathrm{PPh}_{3}\right)_{3}$. Both reactions proceed via characteristic rhodacyclic intermediates.

\section{Acknowledgements}

This work was supported in part by a Grant-in-Aid for Scientific Research (B) (No. 20350048) from the Ministry of Education, Culture, Sports, Science and Technology, Japan. T.K. acknowledges financial support from Yazaki Memorial Foundation for Science and Technology. This research was conducted in part at the Advanced Research Institute (the Research Project of Engineering for Sustainable Environment), Katsura-Int'tech Center, Graduate School of Engineering, Kyoto University.

\section{References and Notes}

1. Patai, S., Ed. The Chemistry of Functional Groups: The Chemistry of Ketenes, Allenes, and Related Compounds Part. 1; John Wiley \& Sons: New York, NY, USA, 1980.

2. Okumoto, H. Handbook of Organopalladium Chemistry for Organic Synthesis; Negishi, E., Ed.; John Wiley \& Sons: New York, NY, USA, 2002; pp. 2655-2662.

3. Ulrich, H. Cumulenes in Click Reactions; John Wiley \& Sons: Chichester, UK, 2009. 
4. Geoffroy, G.L.; Bassner, S.L. Interaction of ketenes with organometallic compounds: Ketene, ketenyl, and ketenylidene complexes. Adv. Organomet. Chem. 1988, 28, 1-83.

5. Xu, Y.-C.; Challener, C.A.; Dragisich, V.; Brandvold, T.A.; Peterson, G.A.; Wulff, W.D.; Willard, P.G. The generation of 2-vinylcyclopentene-1,3-diones via a five-component coupling in the coordination sphere of chromium. J. Am. Chem. Soc. 1989, 111, 7269-7271.

6. Grotjahn, D.B.; Lo, H.C. Fragmentation of 2-pyridyl esters gives both $\eta^{2}(\mathrm{C}, \mathrm{O})-$ and $\eta^{2}(\mathrm{C}, \mathrm{C})$ bound ketene ligands on $\mathrm{C} \operatorname{Ir}\left[\mathrm{P}(i-\mathrm{Pr})_{3}\right]_{2}$. Organometallics 1995, 14, 5463-5465.

7. Lo, H.C.; Grotjahn, D.B. Selective C-C bond formation on the first ketene-alkyne complexes. $J$. Am. Chem. Soc. 1997, 119, 2958-2959.

8. Grotjahn, D.B.; Bikzhanova, G.A.; Hubbard, J.L. Phosphine loss from bis(phosphine)rhodium(I) $\eta^{2}-(C, O)$-diphenylketene complexes leading to $\eta^{4}-\left(C_{4}\right)$ coordination and fluxionality of the ketene. Organometallics 1999, 18, 5614-5619.

9. Grotjahn, D.B.; Collins, L.S.B.; Wolpert, M.; Bikzhanova, G.A.; Lo, H.C.; Combs, D.; Hubbard, J.L. First direct structural comparison of complexes of the same metal fragment to ketenes in both C,C- and C,O-Bonding modes. J. Am. Chem. Soc. 2001, 123, 8260-8270.

10. Grotjahn, D.B.; Hoerter, J.M.; Hubbard, J.L. Double $\mathrm{C}-\mathrm{H}$ activation during functionalization of phenyl(methyl)ketene on iridium(I) using alkynes. Synthesis of 1,4-dien-3-ones. J. Am. Chem. Soc. 2004, 126, 8866-8867.

11 Kondo, T.; Kaneko, Y.; Taguchi, Y.; Nakamura, A.; Okada, T.; Shiotsuki, M.; Ura, Y.; Wada, K.; Mitsudo, T. Rapid ruthenium-catalyzed synthesis of pyranopyrandiones by reconstructive carbonylation of cyclopropenones involving $\mathrm{C}-\mathrm{C}$ bond cleavage. J. Am. Chem. Soc. 2002, 124, 6824-6825.

12 Kondo, T.; Taguchi, Y.; Kaneko, Y.; Niimi, M.; Mitsudo, T. Ru- and Rh-catalyzed C-C bond leavage of cyclobutenones: Reconstructive and selective synthesis of 2-pyranones, cyclopentenes, and cyclohexenones. Angew. Chem. Int. Ed. 2004, 43, 5369-5372.

13 Kondo, T.; Niimi, M.; Nomura, M.; Wada, K.; Mitsudo, T. Rhodium-catalyzed rapid synthesis of substituted phenols from cyclobutenones and alkynes or alkenes via $\mathrm{C}-\mathrm{C}$ bond cleavage. Tetrahedron Lett. 2007, 48, 2837-2839.

14 Hong, P.; Yamazaki, H.; Sonogashira, K.; Hagihara, N. Rhodium carbonyl cluster catalyzed addition of arenes to diphenylketene and aryl isocyanate. Chem. Lett. 1978, 535-538.

15. Yamazaki, H.; Hong, P. Organic reactions with rhodium carbonyl cluster catalysts. J. Mol. Catal. 1983, 21, 133-150.

16 Mitsudo, T.; Kadokura, M.; Watanabe, Y. Palladium complex catalyzed reaction of diphenylketene with terminal acetylenes giving disubstituted acetylenes. Tetrahedron Lett. 1985, 26, 3697-3698.

17. Mitsudo, T.; Kadokura, M.; Watanabe, Y. Palladium complex catalyzed synthesis of $\alpha, \beta$ unsaturated ketones from alkylphenylketene and aroyl chloride. Tetrahedron Lett. 1985, 26, 5143-5144.

18. Mitsudo, T.; Kadokura, M.; Watanabe, Y. Palladium-catalysed reactions of ketenes with allyl acetates or allyl carbonates: novel syntheses of 1,3-dienes and allylated esters. J. Chem. Soc., Chem. Commun. 1986, 1539-1541. 
19. Mitsudo, T.; Kadokura, M.; Watanabe, Y. Palladium-complex-catalyzed reactions of ketenes with allylic carbonates or acetates. Novel syntheses of $\alpha$-allylated carboxylic esters and 1,3-dienes. $J$. Org. Chem. 1987, 52, 1695-1699.

20. Mitsudo, T.; Kadokura, M.; Watanabe, Y. Novel synthesis of $\alpha, \beta$-unsaturated ketones by the palladium-catalyzed arylation of ketenes with aroyl chlorides or the decarbonylative crosscondensation of acyl halides. J. Org. Chem. 1987, 52, 3186-3192.

21. Kondo, T.; Tokoro, Y.; Ura, Y.; Wada, K.; Mitsudo, T. Rhodium-Catalyzed decarbonylative coupling reactions of diphenylketene with alkenes. ChemCatChem 2009, 1, 82-84.

22. Straus, D.A.; Grubbs, R.H. Preparation and reaction of metal-ketene complexes of zirconium and titanium. J. Am. Chem. Soc. 1982, 104, 5499-5500.

23. Huffman, M.A.; Liebeskind, L.S. Insertion of ( $\eta^{5}$-indeny)cobalt(I) into cyclobutenones: the first synthesis of phenols from isolated vinylketene complexes J. Am. Chem. Soc. 1990, 112, 8617-8618.

24. Anderson, B.A.; Wulff, W.D.; Rheingold, A.L. Characterization of the first $\eta^{4}$-vinylketene metal complex from the reaction of a Group 6 Fischer carbene complex and an alkyne. J. Am. Chem. Soc. 1990, 112, 8615-8616.

25. Bao, J.; Wulff, W.D.; Dragisich, V.; Wenglowsy, S.; Ball, R.S. Three-component intramolecular two-alkyne annulations of Fischer carbene complexes: new strategies for steroid synthesis. J. Am. Chem. Soc. 1994, 116, 7616-7630.

26. Brady, W.T. Halogenated ketenes: valuable intermediates in organic synthesis. Synthesis 1971, 415-422.

27. Allen, A.D.; Baigrie, L.M.; Gong, L.; Tidwell, T.T. Cyclopropylketenes: preparation and nucleophilic additions. Can. J. Chem. 1991, 69, 138-145.

28. Osborn, J.A.; Jardine, J.F.; Young, J.F.; Wilkinson, G. Tris(triphenylphosphine)halorhodium(I). Inorg. Synth. 1967, 10, 67-71.

29. McCleverty, J.A.; Wilkinson, G. Chlorocarbonylbis(triphenylphosphine)rhodium and chlorocarbonylbis(triphenylarsine)rhodium. Inorg. Synth. 1966, 8, 214-217.

30. Ahmad, N.; Levinson, J.J.; Robinson, S.D.; Uttley, M.F. Hydrido phosphine complexes of rhodium(I). Inorg. Synth. 1990, 28, 81-83.

31. Wonchoba, E.R.; Parshall, G.W. Complexes of ruthenium, osmium, rhodium, and iridium containing hydride carbonyl, or nitrosyl ligands. Inorg. Synth. 1974, 15, 45-64.

32. Ent, A.; Onderdelinden, A.L. Di- $\mu$-chlorotetrakis(ethylene)dirhodium(I), 2,4-pentanedionatobis(ethylene)rhodium(I), and di- $\mu$-chlorotetracarbonyldirhodium(I). Inorg. Synth. 1974, 15, 14-18.

33. Hallman, P.S.; Stephenson, T.A.; Wilkinson, G. Tetrakis(triphenylphosphine)dichlororuthenium(II) and tris(triphenylphosphine)dichlororuthenium(II). Inorg. Synth. 1970, 12, 237-240.

34. Young, R.; Wilkinson, G. Dihydridotetrakis(triphenylphosphine)ruthenium(II). Inorg. Synth. 1977, $17,75-77$.

Sample Availability: Samples of the compounds 3a-d and 4a-e are available from the authors.

(C) 2010 by the authors; licensee MDPI, Basel, Switzerland. This article is an Open Access article distributed under the terms and conditions of the Creative Commons Attribution license (http://creativecommons.org/licenses/by/3.0/). 